\title{
Alterações da Rigidez Arterial em Pacientes com Estenose Aórtica Grave Submetidos à Cirurgia de Troca Valvar
}

\author{
Arterial Stiffness Changes in Severe Aortic Stenosis Patients Submitted to Valve Replacement Surgery
}

Renata Raimundo, ${ }^{10}$ Francisca Saraiva, ${ }^{1}$ Raquel Moreira, ${ }^{1}$ Soraia Moreira, ${ }^{1}$ Ana Filipa Ferreira, ${ }^{1}$ Rui J. Cerqueira, ${ }^{1,2}{ }^{\circledR 0}$ Mario Jorge Amorim, 1,2 Paulo Pinho, ${ }^{2}$ António Sousa Barros, ${ }^{1}$ André P. Lourenço, ${ }^{1,3}$ Adelino Leite-Moreira ${ }^{1,2}$

Cardiovascular Research and Development Center, Department of Surgery and Physiology, Faculty of Medicine of the University of Porto, ${ }^{1}$

Porto - Portugal

Department of Cardiothoracic Surgery, Centro Hospitalar Universitário São João, ${ }^{2}$ Porto - Portugal

Department of Anesthesiology, Centro Hospitalar Universitário São João, ${ }^{3}$ Porto - Portugal

\section{Resumo}

Fundamento: Pouco se sabe sobre o impacto da estenose aórtica (EA) grave na rigidez aórtica e se ocorre alguma alteração após a remoção da barreira de EA com a cirurgia de substituição da válvula aórtica (SVA).

Objetivo: Estimar as mudanças na velocidade de onda de pulso carotídeo-femoral (VOP) após a cirurgia de SVA e definir os preditores de VOP alta em pacientes com EA grave.

Métodos: Estudo de coorte retrospectivo unicêntrico, incluindo pacientes com EA grave submetidos à cirurgia de SVA com bioprótese, entre fevereiro de 2017 e janeiro de 2019, e medições da VOP (Complior $\left.{ }^{\circledR}\right)$ antes e depois do procedimento ( $2 \pm 1$ meses). Antes e depois da SVA, os valores da VOP foram comparados por meio de testes pareados. foram analisadas as associações de VOP com dados clínicos, bem como aplicados modelos de regressão linear multivariada para estimar os preditores independentes da VOP pré- e pós-operatória. O nível de significância foi estabelecido em $5 \%$.

Resultados: Foram incluídos na amostra 150 pacientes, com média de idade de $72 \pm 8$ anos, sendo $51 \%$ deles do sexo masculino. Identificamos um aumento estatisticamente significativo nos valores de VOP após a cirurgia $(9,0 \pm 2,1 \mathrm{~m} / \mathrm{s}$ vs. 9,9 $\pm 2,2, p<0,001$, antes e depois da SVA, respectivamente) e uma associação inversa com as variáveis de gravidade da EA. No modelo de regressão linear multivariada, idade e pressão arterial sistólica (PAS) foram estabelecidas como preditores independentes da VOP pré- e pós-operatória mais alta, enquanto o gradiente valvar médio mais alto foi considerado um determinante da VOP pré-SVA mais baixa.

Conclusão: Identificamos uma correlação inversa da rigidez arterial com a gravidade da EA em pacientes acometidos, $\mathrm{e}$ um aumento significativo nos valores da VOP após a cirurgia de SVA. Idade avançada e PAS elevada foram associadas a valores mais altos da VOP, embora as medidas de função arterial estivessem dentro da normalidade. (Arq Bras Cardiol. $2021 ; 116(3): 475-476)$

Palavras-chave: Valva Aórtica/cirurgia; Estenose da Valva Aórtica/cirurgia; Substituição da Valva Aórtica/métodos; Análise de Onda de Pulso.

\begin{abstract}
Background: Little is known about the impact of severe aortic stenosis (AS) in aortic stiffness and if there is any change after removing AS barrier with aortic valve replacement (AVR) surgery.
\end{abstract}

Objective: To estimate carotid-femoral pulse wave velocity (PWV) changes after AVR surgery and to define PWV predictors in severe AS patients.

Methods: Single-center retrospective cohort, including patients with severe AS who underwent AVR surgery with bioprostheses, between February 2017 and January 2019 and performed PWV measurements (Complior $®$ ) before and after the procedure ( $2 \pm 1$ months). Before and after AVR, PWV values were compared through paired tests. The associations of PWV with clinical data were studied and multivariate linear regression models were applied to estimate pre and postoperative PWV independent predictors. The significance level was set at 5\%.

Correspondência: Renata Melo Raimundo •

Faculdade de Medicina da Universidade do Porto - Departamento de Cirurgia e Fisiologia - Alameda Prof. Hernâni Monteiro Porto $4200-319$ - Portugal E-mail: rmrcardio@gmail.com

Artigo recebido em 27/08/2019, revisado em 22/11/2019, aceito em 27/12/2019

DOI: https://doi.org/10.36660/abc.20190577 
Results: We included 150 patients in the sample, with mean age of $72 \pm 8$ years, and 51\% being males. We found a statistically significant

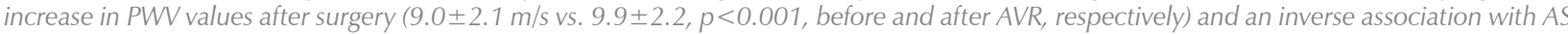
severity variables. In the multivariate linear regression model, age and systolic blood pressure (SBP) were established as independent predictors of higher pre- and postoperative PWV, while higher mean valvular gradient emerged as a determinant of lower pre-AVR PWV.

Conclusion: We documented an inverse correlation of arterial stiffness with the severity of AS in patients with AS, and a significant increase in PWV values after AVR surgery. Advanced age and higher SBP were associated with higher PWV values, although arterial function measurements were within the normal range. (Arq Bras Cardiol. 2021; 116(3):475-476)

Keywords: Aortic Valve/surgery; Aortic Valve Stenosis/surgery; Aortic Valve, Replacement /methods; Pulse Wave Analysis.

Full texts in English - http://www.arquivosonline.com.br

\section{Introdução}

A estenose aórtica degenerativa (EA) é a valvulopatia cardíaca (VPC) mais prevalente em países desenvolvidos e a mais comumente adquirida no mundo, sendo moderada ou grave em $5 \%$ dos pacientes com mais de 75 anos nos EUA. ${ }^{1}$ O tratamento padrão-ouro para sua forma sintomática grave é a substituição da válvula aórtica (SVA). ${ }^{2-4}$

O processo degenerativo da válvula aórtica (VA), que resulta em EA, apresenta alterações fisiopatológicas semelhantes ao processo aterosclerótico responsável pelo aumento da rigidez arterial. ${ }^{5}$ A comunidade científica, portanto, é levada a postular que, na EA, algum grau de disfunção vascular e, consequentemente, rigidez arterial também podem estar presentes. ${ }^{6}$

O envelhecimento também contribui para o enrijecimento vascular, aumentando a pressão aórtica. A medida da velocidade de onda de pulso aórtica (VOP) é o método padrão-ouro, não invasivo e mais reprodutível para avaliar a rigidez arterial. ${ }^{7}$ Basicamente, avalia a capacidade de recuo elástico da aorta, que é diminuída em uma aorta mais rígida, traduzindo-se em um valor de VOP mais alto. ${ }^{8-}$ 10 Portanto, em um paciente com EA, espera-se uma VOP pré-operatória mais elevada, que pode ser recuperada após a SVA. No entanto, alguns estudos têm mostrado que essa associação pode não ser linear, ${ }^{11}$ ou seja, mesmo após o procedimento que alivia a obstrução valvar, uma medida de VOP elevada pode ser observada (ou até maior do que a da avaliação pré-intervenção), representando um aumento na carga vascular. ${ }^{12}$

Há uma forte associação entre uma VOP mais alta e hipertensão sistólica, bem como outros fatores de risco cardiovascular (CV) e doença aterosclerótica. ${ }^{13,14}$ A VOP também tem sido sugerida para prever eventos $\mathrm{CV}$ fatais e não fatais, como acidente vascular cerebral ou síndromes aórticas e coronárias. 8,9,15,16

Há evidências conflitantes em relação às alterações da função arterial após SVA e se a VOP pode ser um marcador de gravidade de EA ou não. Tendo isso em vista, este estudo teve como objetivo esclarecer essa associação.

\section{Objetivo}

O objetivo principal deste estudo foi avaliar a rigidez arterial antes e após a cirurgia de SVA em pacientes com EA grave, usando um equipamento de medição da VOP. Também objetivamos identificar os preditores de resultados da VOP pré- e pós-operatórias nesses pacientes.

\section{Métodos}

\section{Desenho do Estudo e Pacientes}

Estudo retrospectivo unicêntrico, incluindo 150 pacientes com EA grave submetidos à cirurgia SVA com biopróteses entre fevereiro de 2017 e janeiro de 2019, com medições de VOP pré- e pós-operatórias. Pacientes com regurgitação aórtica moderada ou grave concomitante ou procedimentos múltiplos foram excluídos.

\section{Coleta de Dados e Variáveis}

Dados pré-operatórios, cirúrgicos e pós-operatórios foram coletados de prontuários médicos e bancos de dados. Em relação às variáveis pré-operatórias, além dos valores de VOP, também coletamos dados sobre pressão arterial, informações demográficas, fatores de risco cardiovascular, terapia médica em andamento, estado funcional, sintomas e ecocardiograma transtorácico. Cross-clamp (XCT) e tempo de circulação extracorpórea (TCEC), etiologia da doença valvar aórtica e tipo de prótese foram as principais variáveis cirúrgicas. As variáveis de seguimento foram os resultados do

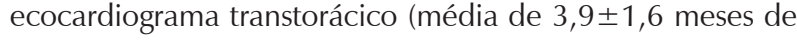
seguimento) e a avaliação da VOP.

O comitê de ética local aprovou o estudo, e todos os dados foram anônimos para análise.

\section{Medição da VOP}

Um método não invasivo (Complior ${ }^{\circledR}$ Analyze) foi usado para avaliar a rigidez arterial por meio da VOP carotídeofemoral antes e depois da cirurgia de SVA. Após alguns minutos em posição supina de repouso, para estabilizar a frequência cardíaca e a pressão arterial, a pressão arterial foi avaliada por meio de um esfigmomanômetro padrão. A distância carótidofemoral foi medida com fita métrica e os dados foram digitados em software específico. Os sensores femoral e carotídeo foram mantidos até que o software atingisse linhas estabilizadas e a melhor qualidade de sinal (acima de 90\%). Cada paciente foi submetido a pelo menos duas medições de VOP em cada sessão. Essas medições foram feitas na admissão dos pacientes no Departamento de Cirurgia Cardiotorácica, no dia anterior ou no mesmo dia da cirurgia. A avaliação pós-operatória ocorreu em média 2,2 $\pm 1,4$ meses após a cirurgia.

\section{Análise Estatística}

A distribuição de dados contínuos foi verificada por meio da análise visual dos histogramas e confirmada pelo teste de 
Shapiro-Wilk. Variáveis contínuas são apresentadas como média e desvio-padrão. As variáveis categóricas foram expressadas em frequências absolutas e relativas. O teste t de amostras pareadas foi usado para comparar variáveis contínuas em dois momentos distintos. As correlações entre a VOP e outras variáveis contínuas foram avaliadas por meio do teste de correlação de Pearson. Os valores de VOP foram comparados entre os grupos usando o teste $\mathrm{t}$ independente. Um modelo de regressão linear multivariada foi construído para estimar os preditores da VOP com base em variáveis clínicas relevantes para a avaliação da rigidez arterial. Os pressupostos da regressão foram verificados, os resíduos foram normalmente distribuídos e as variáveis independentes não foram altamente correlacionadas. Foi utilizado nível de significância de 5\%. As análises estatísticas foram realizadas no Statistical Package for the Social Sciences (SPSS) versão 24 e no ambiente de linguagem R versão 3.6 (R Core Team - 2018. R: Uma linguagem e ambiente para computação estatística. $R$ Foundation for Statistical Computing, Viena, Áustria. URL https:// www.R-project.org/. Frank E Harrell Jr - 2019. rms: Estratégias de modelagem de regressão. Pacote $R$ versão 5.1-3.1).

\section{Resultados}

\section{Amostra}

A caracterização da amostra está representada na Tabela 1. A idade média dos sujeitos foi $72 \pm 8$ anos, sendo $51 \%$ deles do sexo masculino. Hipertensão arterial estava presente em 125 pacientes (83\%), dislipidemia em 114 (76\%), diabetes em 52 (35\%) e histórico de tabagismo em 36 (24\%). Quarenta (27\%) pacientes foram admitidos com classe funcional $\geq$ III da New York Heart Association (NYHA). A maioria dos indivíduos (91\%) fazia uso de medicamentos anti-hipertensivos na admissão.

Durante a cirurgia, $12 \%$ dos pacientes tiveram etiologia congênita confirmada (Tabela 2).

\section{VOP Pré- e Pós-operatória}

Observamos um aumento significativo nos valores de VOP pós-operatória, variando de 9,0 $0 \pm 2,1 \mathrm{~m} / \mathrm{s}$ a 9,9 $9 \pm 2,2$ $\mathrm{m} / \mathrm{s}$ após a cirurgia de SVA ( $p<0,001$, Figura $1 \mathrm{~A})$.

\section{Dados de Monitoramento}

Os valores de pressão arterial sistólica (PAS), gradiente médio da válvula aórtica (GMV) e área da válvula aórtica (AVA) antes e depois da cirurgia de SVA estão representados na Figura 1 (Painéis $B, C$ e D, respectivamente).

\section{Associações da VOP}

A Figura 2 mostra a análise univariada considerando associações da VOP com potenciais preditores. A VOP pré- e pós-operatória teve correlações positivas com idade, PAS e pressão arterial média (PAM), mas foi inversamente associada com as variáveis de gravidade da estenose aórtica. Não encontramos diferenças na VOP de acordo com gênero, hipertensão arterial, diabetes, tabagismo ou VA bicúspide.

No modelo de regressão linear multivariada, idade e PAS foram preditores independentes de VOP pré-operatória mais alta, enquanto o GVM mais alto foi preditor de VOP
Tabela 1 - Características de base

\begin{tabular}{|c|c|}
\hline Variável & $n=150$ \\
\hline Idade, em anos, média (DP) & $72,5(7,6)$ \\
\hline Sexo masculino, $\mathrm{n}(\%)$ & $77(51,3)$ \\
\hline $\mathrm{NYHA} \geq \mathrm{III}, \mathrm{n}(\%)$ & $40(26,9)$ \\
\hline Hipertensão, n (\%) & $125(83,3)$ \\
\hline Atualmente em uso de medicamentos anti-hipertensivos (\%) & $136(90,7)$ \\
\hline Diabetes, n (\%) & $52(34,9)$ \\
\hline Dislipidemia, n (\%) & $114(76,0)$ \\
\hline Histórico de tabagismo, $\mathrm{n}(\%)$ & $36(24,0)$ \\
\hline Índice de massa corpórea, kg/m², média (DP) & $28,6(4,3)$ \\
\hline Obesidade (IMC $\left.\geq 30.00 \mathrm{~kg} / \mathrm{m}^{2}\right), \mathrm{n}(\%)$ & $55(36,7)$ \\
\hline Doença arterial coronária, n (\%) & $19(12,7)$ \\
\hline Arteriopatia extracardíaca, n (\%) & $22(14,7)$ \\
\hline Doença renal crônica (DC < 85ml/min), n (\%) & $87(58,0)$ \\
\hline Depuração de creatinina (DC), ml/min, média (DP) & $83,7(29,7)$ \\
\hline \multicolumn{2}{|c|}{$\begin{array}{l}\text { IMC: indice de massa corpórea; min: minuto; NYHA: New York Heart } \\
\text { Association; DP: desvio-padrão. Arteriopatia extracardiaca foi considerada } \\
\text { se o paciente tinha claudicação, oclusão carotídea ou estenose }>50 \% \text {, } \\
\text { amputação devido a doença arterial, intervenção anterior ou planejada } \\
\text { na aorta abdominal, artérias dos membros ou carótidas, ou histórico de } \\
\text { acidente vascular cerebral. A doença arterial coronariana foi definida } \\
\text { quando os pacientes haviam sido submetidos a intervenção coronária } \\
\text { percutânea no passado, ou tinham estenose coronariana }>50 \% \text {, mas sem } \\
\text { indicação de cirurgia. }\end{array}$} \\
\hline
\end{tabular}

pré-operatória mais baixa (Tabela 3 e Figura 3). Idade e PAS foram considerados preditores independentes de VOP pósoperatória mais alta (Tabela 4 e Figura 4).

\section{Discussão}

Este estudo retrospectivo mostrou uma correlação inversa da rigidez arterial com a gravidade da EA e um aumento significativo nos valores de VOP após a cirurgia de SVA em pacientes com EA grave. Idade avançada e PAS elevada foram associadas a valores mais altos de VOP, embora as medidas de função arterial estivessem dentro da normalidade.

A VOP reflete o enrijecimento arterial devido à perda das propriedades elásticas funcionais da aorta. Trata-se de uma das primeiras manifestações de dano estrutural reversível à parede do vaso, e a VOP é considerada uma técnica com aplicabilidade clínica tanto para identificar quanto para estratificar doenças cardiovasculares. ${ }^{8,16}$

Avaliar o impacto da EA degenerativa na árvore arterial permanece um desafio, uma vez que os mecanismos subjacentes à interação da função vascular e valvar continuam quase desconhecidos. ${ }^{17}$

Liu, et al. ${ }^{5}$ mostraram associação do aumento da VOP com maior escore de cálcio na VA. ${ }^{5}$ Esses resultados sugerem que a deposição de cálcio é uma via importante no processo degenerativo da válvula e da parede aórtica. Além disso, mostraram associação entre aumento da VOP e gradiente de pressão AV avaliado por ecocardiografia. Alterações fisiopatológicas semelhantes são provavelmente compartilhadas 
entre a degeneração da VA e o enrijecimento das grandes artérias. Korkmaz et al., ${ }^{18}$ estimaram o enrijecimento arterial usando o Índice Tornozelo-Braquial (ITB) e encontrou maior rigidez arterial em pacientes com esclerose da válvula aórtica, ${ }^{18}$ sugerindo que a degeneração da VA e o enrijecimento das grandes artérias poderiam ter alterações fisiopatológicas semelhantes. De fato, Emir Cantuk et al., ${ }^{11}$ relataram uma associação significativa entre a gravidade da EA e o aumento da VOP. ${ }^{11}$ Por outro lado, nosso estudo mostra uma correlação inversa entre os gradientes da VA e a VOP pré-operatória. Isso pode ser parcialmente explicado pela obstrução que pode influenciar nas medidas das propriedades arteriais, mascarando os reais efeitos da carga vascular na aorta. El-Chilali et al, ${ }^{6}$ que estudaram pacientes mais velhos (>70 anos) com EA grave e mediram a VOP de forma invasiva, observaram o mesmo: uma correlação inversa entre GVM e VOP. ${ }^{6}$ Essa hipótese é reforçada pelo aumento da VOP observado após a cirurgia de SVA.

Existe um número limitado de estudos que avaliaram a função vascular aórtica em pacientes com EA grave após a intervenção até o momento. ${ }^{11,19,20}$ Canturk et al., ${ }^{11}$ não encontraram diferenças significativas na VOP após a cirurgia. ${ }^{11}$ Por outro lado, Nemes et al.. ${ }^{20}$ demonstraram melhora da função vascular um ano após a SVA. ${ }^{20}$ Em nosso estudo, a VOP aumentou significativamente após a cirurgia de SVA. Alguns

Tabela 2 - Dados pré-operatórios

\begin{tabular}{lc}
\hline Variável & $\mathrm{n}=150$ \\
\hline Etiologia da válvula aórtica, $\mathrm{n}(\%)$ & \\
\hline Degenerativa & $132(88,0)$ \\
\hline Congênita & $18(12,0)$ \\
\hline Tempo de CEC, minutos, média (DP) & $78(26)$ \\
\hline Tempo de clamp aórtico, minutos, média (DP) & $57(20)$ \\
\hline
\end{tabular}

CEC: circulação extracorpórea; DP: desvio-padrão. mecanismos são considerados para explicar esse resultado: o alívio da obstrução valvar após a SVA leva a árvore arterial a operar em um nível de pressão mais elevado, aumentando a carga vascular. Inclusive, Yotti et al. ${ }^{19}$ mostraram que o alívio da obstrução do fluxo de saída aumenta imediatamente as pressões arteriais e a impedância vascular, induzindo um comportamento vascular mais rígido. ${ }^{19}$ De maneira geral, esses achados refletemse na prática clínica, uma vez que é comum iniciar tratamento com anti-hipertensivos após a correção da EA, seja por implante de válvula aórtica transcateter (TAVI) ou por cirurgia. ${ }^{19,21,22}$

Outra explicação, apontada por Barbetseas,et al., ${ }^{12}$ seria que o aumento da rigidez arterial após a cirurgia de SVA ocorre devido à lesão da parede aórtica e destruição de vasa vasorum, bem como alteração da composição das fibras da parede aórtica, resultando em enrijecimento aórtico. No entanto, devemos reforçar que essa diminuição da distensibilidade aórtica foi avaliada uma semana após a SVA, enquanto seis meses após a cirurgia, a função aórtica melhorou, atingindo níveis semelhantes aos pré-operatórios. ${ }^{12}$ Isso pode representar um efeito transitório descrito pelos autores como "atordoamento da raiz aórtica". ${ }^{23}$

Um estudo que comparou TAVI e SVA mostrou alterações significativas na VOP apenas em pacientes cirúrgicos, sugerindo que, em pacientes submetidos à TAVI, as propriedades elásticas são mantidas porque não há manipulação cirúrgica. ${ }^{24}$

Em nosso estudo, a VOP também apresentou associação significativa com a idade, que foi considerada um preditor independente de valores mais elevados de VOP. A alteração da VOP em idosos já está bem estabelecida na literatura. ${ }^{8,17,25}$ Estudos mostram um aumento da VOP com o envelhecimento, provavelmente relacionado à dilatação e enrijecimento da aorta, uma vez que a impedância aumenta e a complacência arterial diminui com o envelhecimento. ${ }^{8,26}$ Nosso estudo dá suporte a esses resultados, sendo que idade foi fortemente correlacionada com a VOP pré- e pós-operatória.

A hipertensão arterial sistêmica também se mostrou um preditor de aumento da $\mathrm{VOP}^{9,27}$ o que está de acordo com nossos resultados, uma vez que PAS elevada foi associada a à VOP

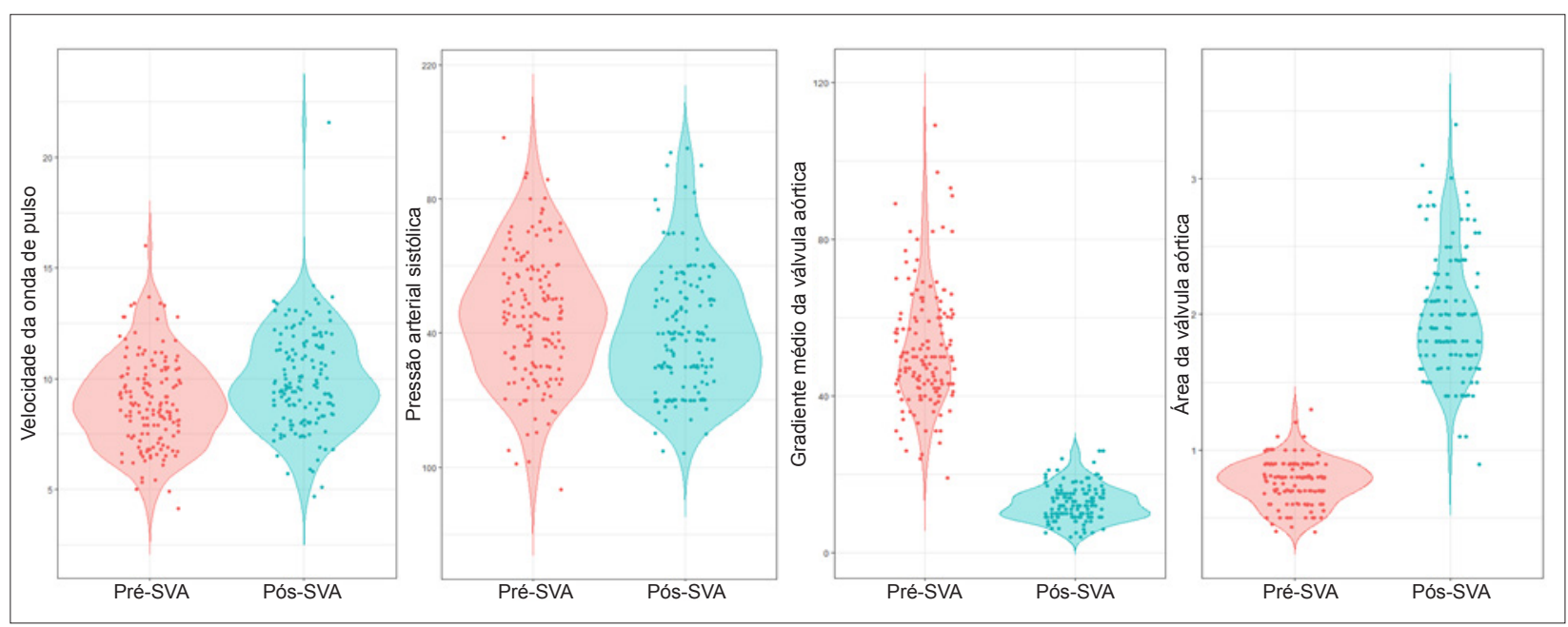

Figura 1 - Gráficos em violino dos valores pré- (vermelho) e pós-operatório (azul) da velocidade da onda de pulso (A), pressão arterial sistólica (B), gradiente médio da válvula aórtica $(C)$ e área da válvula aórtica $(D)$. 


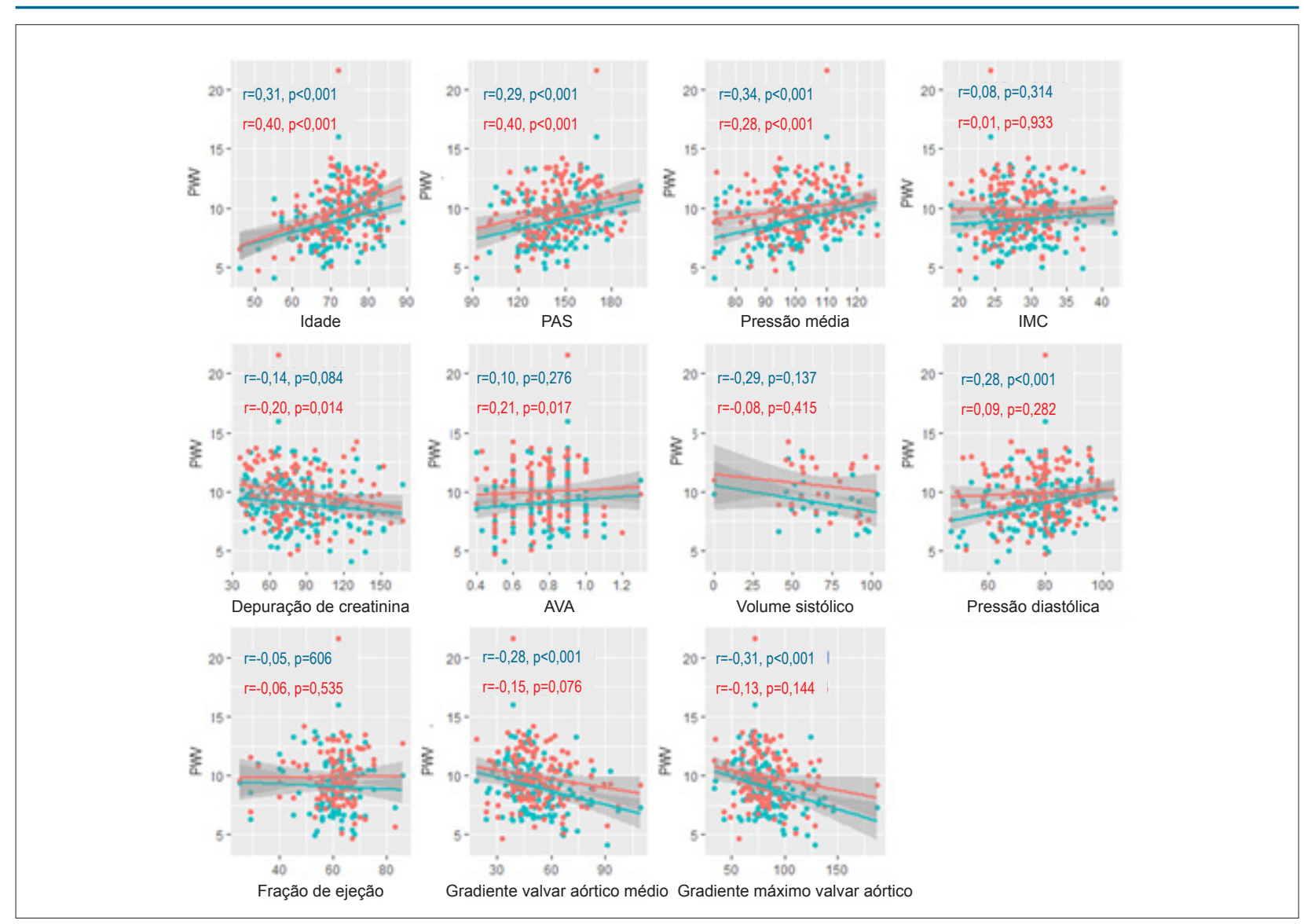

Figura 2 - Gráficos de dispersão das relações pré-operatórias (azul) e pós-operatórias (vermelho) da VOP com idade, PAS: pressão arterial sistólica, pressão média; IMC: índice de massa corpórea, depuração de creatinina; AVA: área da válvula aórtica, volume sistólico, pressão diastólica, fração de ejeção, gradiente valvar aórtico médio e gradiente máximo valvar aórtico.

Tabela 3 - Resumo da análise de regressão multivariada (variável dependente: VOP pré-operatória)

\begin{tabular}{|c|c|c|c|c|}
\hline Variável & $\beta$ & EP & b & $p$ \\
\hline Intercepção & 0,593 & 2,576 & 0,23 & 0,818 \\
\hline Pressão arterial sistólica & 0,020 & 0,008 & 2,42 & 0,017 \\
\hline Diabetes & 0,428 & 0,344 & 1,24 & 0,215 \\
\hline Depuração de creatinina & $-0,001$ & 0,007 & $-0,14$ & 0,889 \\
\hline Doença arterial coronária & 0,531 & 0,498 & 1,07 & 0,288 \\
\hline IMC & 0,059 & 0,040 & 1,46 & 0,147 \\
\hline Idade & 0,070 & 0,025 & 2,87 & 0,004 \\
\hline Sexo & 0,244 & 0,336 & 0,73 & 0,469 \\
\hline GVM & $-0,029$ & 0,011 & $-2,75$ & 0,007 \\
\hline Válvula aórtica bicúspide & $-0,299$ & 0,498 & $-0,60$ & 0,549 \\
\hline
\end{tabular}

IMC: índice de massa corpórea; $\beta$ : coeficiente de regressão não padronizado; b: coeficiente padronizado; GVM: gradiente médio da válvula; EP: erro padrão do coeficiente. 


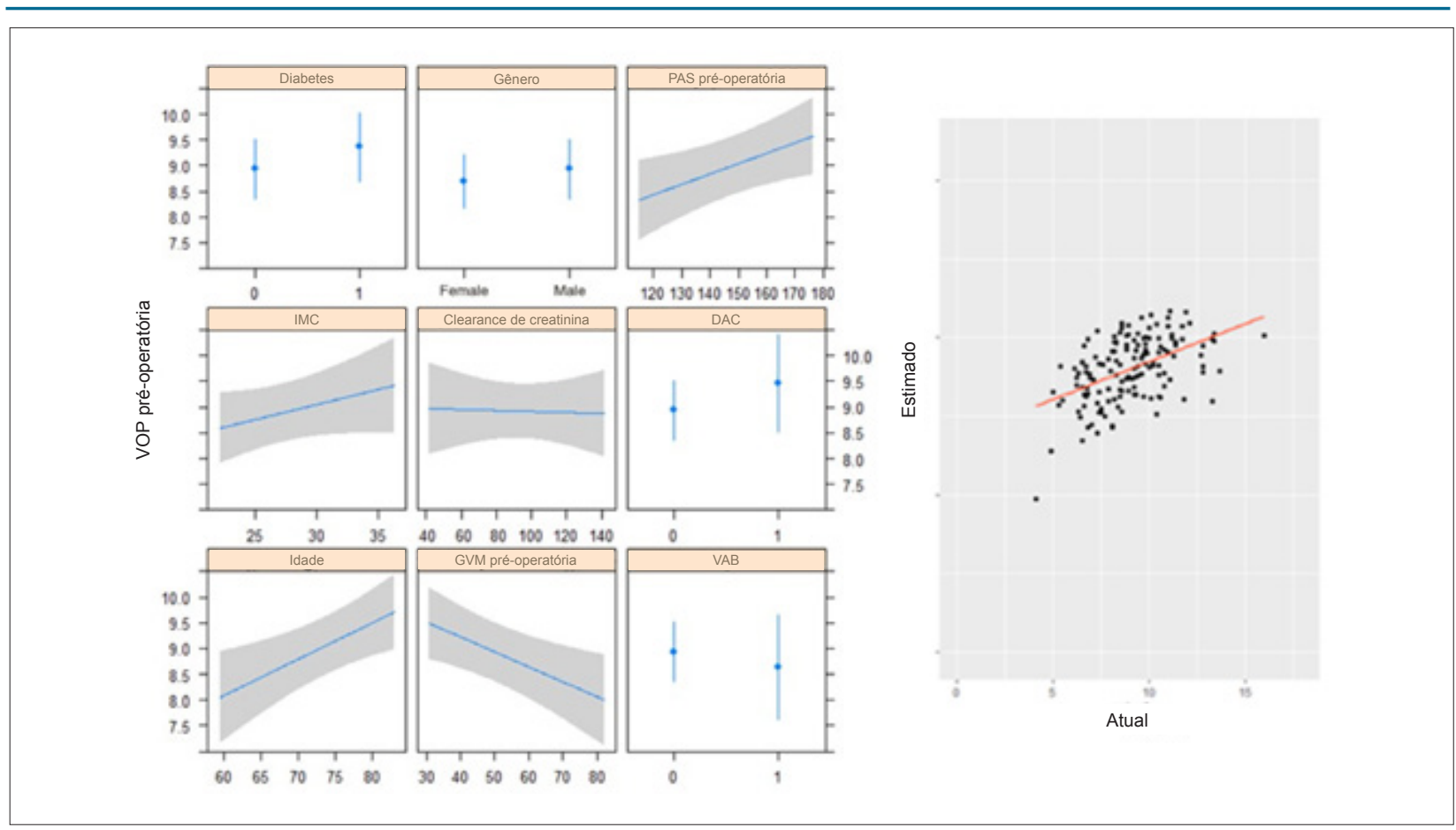

Figura 3 - Modelo de regressão linear multivariada de VOP pré-operatória e valores estimados versus reais. VAB: válvula aórtica bicúspide; DAC: doença arterial coronariana; GVM: gradiente médio da válvula; PAS: pressão arterial sistólica; IMC: índice de massa corpórea.

Tabela 4 - Resumo da análise de regressão multivariada (variável dependente: VOP pós-operatória)

\begin{tabular}{lllll}
\hline Variável & $\boldsymbol{\beta}$ & EP & $\mathbf{b}$ & $\mathbf{p}$ \\
\hline Intercepção & $-2,706$ & 2,391 & $-1,13$ & 0,260 \\
\hline Pressão arterial sistólica & 0,037 & 0,009 & 4,11 & $<0,001$ \\
\hline Diabetes & 0,448 & 0,352 & 1,27 & 0,205 \\
\hline Doença arterial coronária & 0,837 & 0,508 & 1,65 & 0,102 \\
\hline IMC & 0,023 & 0,040 & 0,58 & 0,564 \\
\hline Idade & 0,097 & 0,023 & 4,13 & $<0,001$ \\
\hline Sexo & 0,287 & 0,351 & 0,82 & 0,415 \\
\hline GVM & $-0,056$ & 0,038 & $-1,46$ & 0,146 \\
\hline
\end{tabular}

IMC: índice de massa corpórea; $\beta$ : coeficiente de regressão não padronizado; b: coeficiente padronizado; GVM: gradiente médio da válvula; EP: erro padrão do coeficiente.

elevada. Como mais de $80 \%$ da nossa amostra tinha hipertensão sistêmica, não encontramos diferenças substanciais entre os pacientes com e sem esse fator de risco, mas reconhecemos que a VOP tem sido amplamente utilizada para fins de estratificação de risco como um fator de risco independente para mortalidade por todas as causas e por doenças cardiovasculares. ${ }^{27,28}$

Considerando que a aterosclerose é comumente associada ao envelhecimento arterial e doença arterial coronariana (DAC), seria de se esperar que pacientes com DAC apresentassem aumento na VOP. ${ }^{29,30}$ De fato, nossos resultados mostraram uma VOP pós-SVA significativamente maior em pacientes com DAC em comparação a pacientes sem DAC. No entanto, essa diferença estava ausente na VOP pré-SVA, o que sustenta a hipótese de um efeito de mascaramento pela EA, atenuando a manifestação de enrijecimento aórtico por DAC.

Estudos anteriores demonstraram disfunção vascular em pacientes obesos, ${ }^{31,32}$ mas, em nosso estudo, o índice de massa corporal (IMC) não foi considerado um fator preditor independente para VOP pré- e pós-operatória elevada.

O impacto estimado da EA na VOP continuará aberto para debate, pois nosso estudo teve várias limitações: 1) estudo unicêntrico; 2) natureza retrospectiva, o que leva à ausência de alguns dados (9\% ausentes na análise multivariada realizada), 


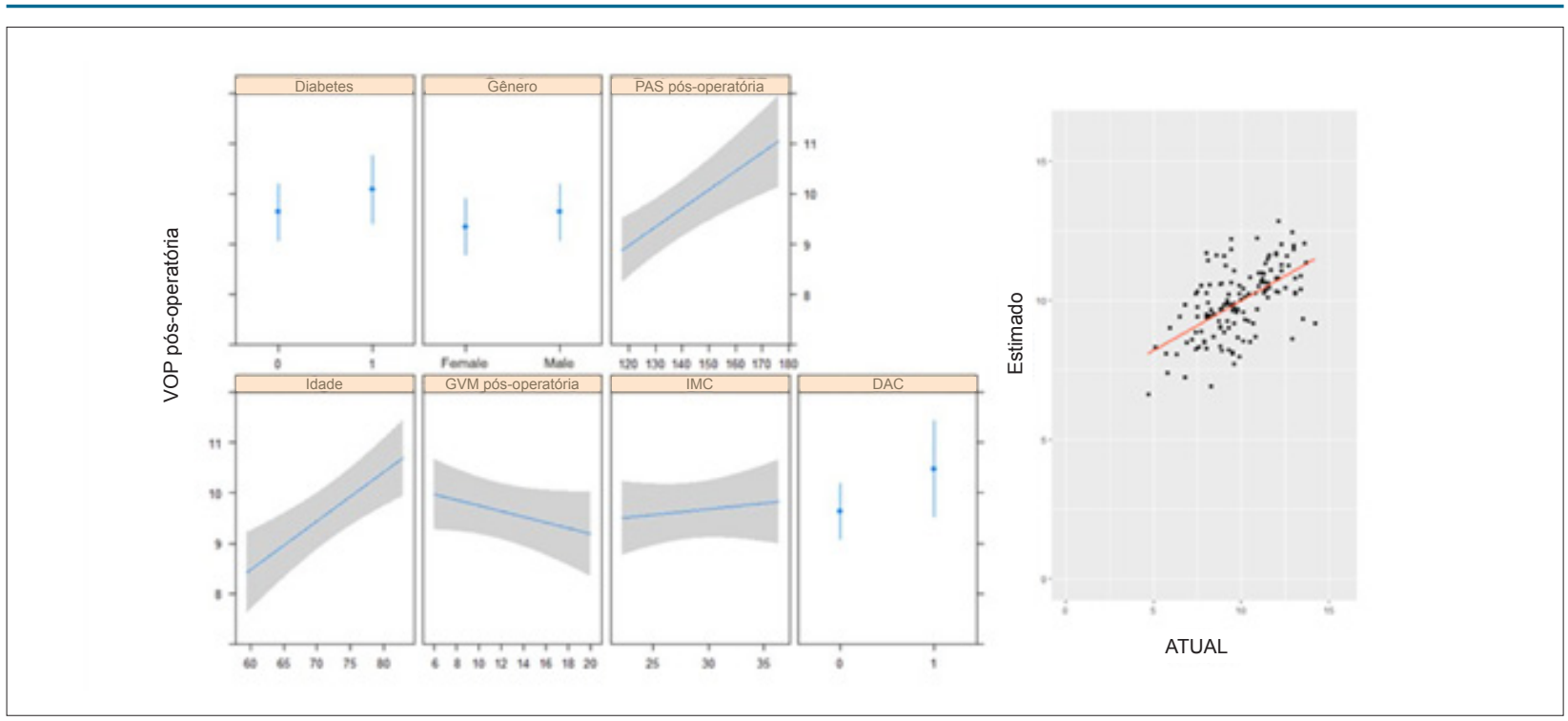

Figura 4 - Modelo de regressão linear multivariada da VOP pós-operatória e valores estimados versus reais. IMC: índice de massa corpórea; DAC: doença arterial coronariana; GVM: gradiente médio da válvula; PAS: pressão arterial sistólica.

medições de VOP pós-operatórias não programadas sistematicamente e realizadas ao mesmo tempo após a cirurgia (2 pacientes cujas cirurgias foram adiadas para $12 \mathrm{e}$ 65 dias após a medição pré-operatório da VOP), e ausência de medição de longo prazo; 3) a seleção da amostra não foi aleatória nem consecutiva e seu tamanho relativamente pequeno limita a generalização externa dos resultados; 4) As medidas de VOP também apresentam limitações, como alta variabilidade de acordo com o estado do paciente; por exemplo, a pressão arterial pode não estar controlada na medição pré-operatória (todos os pacientes em jejum e com descontinuação da terapia farmacológica) em comparação com a pós-operatória (todos os pacientes sem jejum e alguns deles com pressão arterial controlada farmacologicamente).

\section{Conclusão}

Embora alguns estudos sugiram que a rigidez aórtica é aumentada em EA devido a um componente aterosclerótico concomitante, nossos achados sugerem que EA pode atenuar a rigidez arterial real da parede aórtica, já que a gravidade de EA está inversamente relacionada à VOP, enquanto um pequeno aumento na VOP foi observado após a cirurgia de SVA e alívio do gradiente de pressão ventricular-aórtico. Como esperado, idade e PAS foram determinantes independentes de VOP mais alta em pacientes com EA grave e permaneceram os mesmos após a cirurgia. Mais estudos são necessários para fornecer uma melhor compreensão da história natural da EA e sua relação com a função vascular.

\section{Financiamento}

Este trabalho foi apoiado pelo projeto DOCnet (NORTE01-0145-FEDER-000003), pelo Programa Operacional Regional do Norte de Portugal (NORTE 2020), no âmbito do Acordo de Parceria PORTUGAL 2020, pelo Fundo Europeu de Desenvolvimento Regional (FEDER), projeto NETDIAMOND
(POCl-01-0145-FEDER-016385), apoiado pelos Fundos Europeus Estruturais e de Investimento, Programa Operacional Regional de Lisboa 2020 e fundos nacionais da Fundação Portuguesa para a Ciência e Tecnologia. R. Raimundo e F. Saraiva foram apoiados pelo Programa de Bolsas de Doutorado (Norte-08-5369-FSE-000024), financiados pelo Programa Operacional Regional do Norte de Portugal (NORTE 2020), por meio da CCDRN, PORTUGAL 2020 e da Rede Social Europeia Fundo (ESF).

\section{Contribuição dos Autores}

Concepção e desenho da pesquisa: Raimundo R, Saraiva F, Moreira R, Moreira S, Ferreira AF, Cerqueira RJ, Amorim MJ, Lourenço AP, Leite-Moreira A; Obtenção de dados: Raimundo R, Saraiva F, Moreira R, Moreira S, Ferreira AF; Análise e interpretação dos dados e Análise estatística: Raimundo R, Saraiva F, Moreira R, Barros AS, Lourenço AP, Leite-Moreira A; Redação do manuscrito e Revisão crítica do manuscrito quanto ao conteúdo intelectual importante: Raimundo R, Saraiva F, Moreira R, Moreira S, Ferreira AF, Cerqueira RJ, Amorim MJ, Pinho P, Barros AS, Lourenço AP, Leite-Moreira A.

\section{Potencial Conflito de Interesses}

Declaro não haver conflito de interesses pertinentes.

\section{Fontes de Financiamento}

O presente estudo foi financiado parcialmente pelo Projeto Docmet (norte 01-0145 - FEDER, 000031- Netdiamond (POCI 01-0145 - FEDER 016385)

\section{Vinculação Acadêmica}

Este artigo é parte de tese de Doutorado de Renata Melo Raimundo pela Faculdade de Medicina da Universidade do Porto. 


\section{Referências}

1. lung B, Vahanian A. Epidemiology of valvular heart disease in the adult. Nat Rev Cardiol. 2011;8(3):162-72.

2. Genereux P, Stone GW, O'Gara PT, Marquis-Gravel G, Redfors B, Giustino G, et al. Natural History, Diagnostic Approaches, and Therapeutic Strategies for Patients With Asymptomatic Severe Aortic Stenosis. J Am Coll Cardiol. 2016;67(19):2263-88.

3. Baumgartner H, Falk V, Bax JJ, De Bonis M, Hamm C, Holm PJ, et al. 2017 ESC/EACTS Guidelines for the management of valvular heart disease. Eur Heart J. 2017;38(36):2739-91

4. Nishimura RA, Otto CM, Bonow RO, Carabello BA, Erwin JP, 3rd, Fleisher LA, et al. 2017 AHA/ACC Focused Update of the 2014 AHA/ACC Guideline for the Management of Patients With Valvular Heart Disease: A Report of the American College of Cardiology/American Heart Association Task Force on Clinical Practice Guidelines. J Am Coll Cardiol. 2017;70(2):252-89.

5. Liu PY, Tsai WC, Lin CC, Hsu CH, Haung YY, Chen JH. Invasive measurements of pulse wave velocity correlate with the degree of aortic valve calcification and severity associated with matrix metalloproteinases in elderly patients with aortic valve stenosis. Clin Sci (Lond). 2004;107(4):415-22.

6. El-Chilali K, Farouk H, Abdelhafez M, Neumann T, Alotaibi S, Wendt D, et al. Predictors of aortic pulse wave velocity in the elderly with severe aortic stenosis. Aging Clin Exp Res. 2016;28(3):519-25.

7. Vlachopoulos C, Aznaouridis K, Stefanadis C. Prediction of cardiovascular events and all-cause mortality with arterial stiffness: a systematic review and meta-analysis. J Am Coll Cardiol. 2010;55(13):1318-27.

8. Sutton-Tyrrell K, Najjar SS, Boudreau RM, Venkitachalam L, Kupelian V, Simonsick EM, et al. Elevated aortic pulse wave velocity, a marker of arteria stiffness, predicts cardiovascular events in well-functioning older adults. Circulation. 2005;111(25):3384-90.

9. Wang KL, Cheng HM, Sung SH, Chuang SY, Li CH, Spurgeon HA, et al. Wave reflection and arterial stiffness in the prediction of 15-year all-cause and cardiovascular mortalities: a community-based study. Hypertension. 2010;55(3):799-805.

10. Laurent S, Cockcroft J, Van Bortel L, Boutouyrie P, Giannattasio C, Hayoz D, et al. Expert consensus document on arterial stiffness: methodological issues and clinical applications. Eur Heart J. 2006;27(21):2588-605.

11. Canturk E, Cakal B, Karaca O, Omaygenc O, Salihi S, Ozyuksel A, et al. Changes in Aortic Pulse Wave Velocity and the Predictors of Improvement in Arterial Stiffness Following Aortic Valve Replacement. Ann Thorac Cardiovasc Surg. 2017;23(5):248-55.

12. Barbetseas J, Alexopoulos N, Brili S, Aggeli C, Marinakis N, Vlachopoulos $\mathrm{C}$, et al. Changes in aortic root function after valve replacement in patients with aortic stenosis. Int J Cardiol. 2006;110(1):74-9.

13. McEniery CM, Cockcroft JR. Does arterial stiffness predict atherosclerotic coronary events? Adv Cardiol. 2007;44:160-72.

14. Kullo IJ, Malik AR. Arterial ultrasonography and tonometry as adjuncts to cardiovascular risk stratification. J Am Coll Cardiol. 2007;49(13):1413-26.

15. Mattace-Raso FU, van der Cammen TJ, Hofman A, van Popele NM, Bos ML, Schalekamp MA, et al. Arterial stiffness and risk of coronary heart disease and stroke: the Rotterdam Study. Circulation. 2006;113(5):657-63.

16. Mitchell GF, Hwang SJ, Vasan RS, Larson MG, Pencina MJ, Hamburg NM, et al. Arterial stiffness and cardiovascular events: the Framingham Heart Study. Circulation. 2010;121(4):505-11.
17. Celik S, Durmus I, Korkmaz L, Gedikli O, Kaplan S, Orem C, et al. Aortic pulse wave velocity in subjects with aortic valve sclerosis. Echocardiography. 2008;25(10):1112-6.

18. Korkmaz L, Agac MT, Bektas H, Varol MO, Erkan H, Acar Z, et al. Aortic valve sclerosis is a sign of increased arterial stiffness in clinically asymptomatic subjects. Cardiol J. 2013;20(3):318-22.

19. Yotti R, Bermejo J, Gutierrez-Ibanes E, Perez del Villar C, Mombiela T, Elizaga J, et al. Systemic vascular load in calcific degenerative aortic valve stenosis: insight from percutaneous valve replacement. J Am Coll Cardiol. 2015;65(5):423-33.

20. Nemes A, Galema TW, Geleijnse ML, Soliman OI, Yap SC, Anwar AM, et al. Aortic valve replacement for aortic stenosis is associated with improved aortic distensibility at long-term follow-up. Am Heart J. 2007;153(1):147-51

21. Perlman GY, Loncar S, Pollak A, Gilon D, Alcalai R, Planer D, et al. Post-procedural hypertension following transcatheter aortic valve implantation: incidence and clinical significance. JACC Cardiovasc Interv. $2013 ; 6(5): 472-8$

22. Dahl JS, Videbaek L, Poulsen MK, Pellikka PA, Veien K, Andersen LI, et al. Effect of candesartan treatment on left ventricular remodeling after aortic valve replacement for aortic stenosis. Am J Cardiol. 2010;106(5):713-9.

23. Bruschi G, Maloberti A, Sormani P, Colombo G, Nava S, Vallerio P, et al. Arterial Stiffness in Aortic Stenosis: Relationship with Severity and Echocardiographic Procedures Response. High Blood Press Cardiovasc Prev. 2017;24(1):19-27.

24. Musa TA, Uddin A, Fairbairn TA, Dobson LE, Sourbron SP, Steadman CD et al. Assessment of aortic stiffness by cardiovascular magnetic resonance following the treatment of severe aortic stenosis by TAVI and surgical SVA. J Cardiovasc Magn Reson. 2016;18(1):37.

25. Meaume S, Benetos A, Henry OF, Rudnichi A, Safar ME. Aortic pulse wave velocity predicts cardiovascular mortality in subjects $>70$ years of age. Arterioscler Thromb Vasc Biol. 2001;21(12):2046-50.

26. Ohmori K, Emura S, Takashima T. Risk factors of atherosclerosis and aortic pulse wave velocity. Angiology. 2000;51(1):53-60.

27. Ecobici M, Stoicescu C. Arterial Stiffness and Hypertension - Which Comes First? Maedica (Buchar). 2017;12(3):184-90.

28. Bastos JM, Bertoquini S, Polonia J. Prognostic significance of ambulatory arterial stiffness index in hypertensives followed for 8.2 years: its relation with new events and cardiovascular risk estimation. Rev Port Cardiol. 2010;29(9):1287-303.

29. Rodondi N, Marques-Vidal P, Butler J, Sutton-Tyrrell K, Cornuz J, Satterfield S, et al. Markers of atherosclerosis and inflammation for prediction of coronary heart disease in older adults. Am J Epidemiol. 2010;171(5):540-9.

30. Tomiyama H, Koji Y, Yambe M, Shiina K, Motobe K, Yamada J, et al. Brachialankle pulse wave velocity is a simple and independent predictor of prognosis in patients with acute coronary syndrome. Circ J. 2005;69(7):815-22.

31. Nordstrand N, Gjevestad E, Dinh KN, Hofso D, Roislien J, Saltvedt E, et al. The relationship between various measures of obesity and arterial stiffness in morbidly obese patients. BMC Cardiovasc Disord. 2011;11:7.

32. Rider OJ, Tayal U, Francis JM, Ali MK, Robinson MR, Byrne JP, et al. The effect of obesity and weight loss on aortic pulse wave velocity as assessed by magnetic resonance imaging. Obesity (Silver Spring). 2010;18(12):2311-6. 